\title{
Quasinormal Modes of Extended Gravity Black Holes
}

\author{
Abdul Jawad, ${ }^{*}$ Shahid Chaudhary, ${ }^{\dagger}$ and Muhammad Yasir ${ }^{\ddagger}$ \\ Department of Mathematics, COMSATS Institute of \\ Information Technology, Lahore-54000, Pakistan \\ Ali Övgün ${ }^{\S}$ and İzzet Sakallı \\ Physics Department, Eastern Mediterranean University, \\ Famagusta, 99628 North Cyprus, via Mersin 10, Turkey
}

\begin{abstract}
Black hole's quasinormal frequencies are basically the complex numbers which provide information about the relaxation of perturbations and depend on the characteristics of the spacetime and types of perturbations. In this paper, we evaluate the quasinormal modes of Hayward black hole in Einstein Gauss-Bonnet gravity, Hayward black hole in anti-de Sitter space (AdS) spacetime, and 4-dimensional black hole in Einstein-Lovelock gravity. By utilizing the WKB resonance technique, we examine the quasinormal modes frequencies $\omega$ by shifting the charge parameter $Q$ (it is also identified with the cosmological constant), circular harmonic index $l$, and mass of scalar field $m$. We also study the relaxation rate for those black holes and find out that the relaxation rate increases with increasing values of $Q$. We observe that real and imaginary components of the quasinormal modes are not linear functions as similar to Reisnner Nordström-AdS. For large values of charge, quasinormal ringing becomes slower to settle down to thermal equilibrium and hence the frequency of the oscillation becomes smaller.
\end{abstract}

Keywords: Relativity and gravitation, Black Holes, Quasinormal modes, M odified Gravity, Einstein-Gauss-Bonnet Gravity

\section{INTRODUCTION}

In the last few decades, there has been a growing interest in quasinormal modes (QNMs) of black holes (BHs); especially after the detection of the gravitational waves by the LIGO scientists [1], this interest is further increased. The quasinormal ringing influences the most of the phenomenon about the BHs including the perturbation dynamics. In fact, QNMs are unique fingerprints aimed at directly identifying the BH entity. Detection of the QNMs is expected to be perceived during the gravitational wave observations in the near future. To obtain more information from the signals of gravitational waves, it is very important to know how the QNMs act for the parameters of BHs in various models. The theory of QNMs of compact objects from both the mathematical and astrophysical points of view was considerably discussed in the literature (see for example $[2,3]$ and references therein). Some researchers have generalized the effects of dark matter and dark energy on QNMs and some have developed the investigation of QNMs of various type of black holes in lower/higher dimensional spacetimes [4-14]. It is also worth noting that QNMs of BHs in AdS space (when the black hole is immersed in an expanding universe) have been recently studied $[15]$.

Singularity of the $\mathrm{BH}$ is a recognized as trouble in a general relativity (GR). At the singular point, it means that flow will be divergent, so it implies that all the material physics laws come up short at that point. Bardeen $[3,16]$ proposed a regular spacetime with a horizon and without singularity. But the physical source associated with Bardeen's solution was clarified much later, when Ayon-Beato and Garcia [17] interpreted it as the gravitational field of a nonlinear magnetic monopole of self gravitating magnetic field. Such BHs in general are known as regular BHs. Their metrics and curvature are all regular everywhere.

Hayward [18] also derived regular spacetimes, which describe the formation of a (locally defined) BH from an initial vacuum region, its quiescence as a static region, and its subsequent evaporation to a vacuum region. The static region of a Hayward $\mathrm{BH}$ is Bardeen-like, supported by finite density and pressures, vanishing rapidly at large radius and behaving as a cosmological constant at small radius. Hayward $\mathrm{BH}$ has attracted extraordinary attention in innumerable studies, like QNM of the Hayward BH proposed by Lin et al. [19]. Furthermore, thin-shell wormhole construction from the Hayward (regular) $\mathrm{BH}$ [20] and their detailed stability analysis [21], and strong deflection

\footnotetext{
*Electronic address: jawadab181@yahoo.com; abduljawad@ciitlahore.edu.pk

${ }^{\dagger}$ Electronic address: shahidpeak00735@gmail.com

${ }^{\ddagger}$ Electronic address: yasirciitsahiwal@gmail.com

$\S$ Electronic address: ali.ovgun@emu.edu.tr; https://www. aovgun.com

IElectronic address: izzet.sakalli@emu.edu.tr
} 
gravitational lensing [22] by a modified Hayward BH [23] are also studied. The Hayward's metric was also considered as a particle accelerator [24].

Our main goal in this study is to study the QNMs for Hayward BH in Einstein-Gauss-Bonnet (EGB) gravity, in AdS spacetime, and in Einstein-Lovelock (EL) gravity. The discontinuance directed toward GR without any problem acquired by taking $\alpha \rightarrow 0$, where $\alpha$ is the parameter that deals with the distinction between GR and Einstein GB gravity. The QNM for BHs in EGB has been considered in [25] and [26], for scalar and tensor modes, separately, and its solidness criteria has been generalized. Likewise, one can have an option to concentrate how the existence of the GB term will adjust the spectra of emanation created by EL theory, inside a BH, as contrasted and a similar system leads to GR. QNMs are conditional on fulfilling the boundary conditions: purely ingoing waves at the event horizon and outgoing waves at spatial infinity [27].

This paper is organized as follows: In Sec. II, we briefly introduce the QNMs. Section III is devoted to Hayward BH of EGB gravity with their spacetime structure. We also represent the relevant computations and graphs. In Sec. IV, we compute the scalar QNM of Hayward BH in AdS spacetime. In Sec. V, we make similar QNM computations for the Hayward BHs in 4D EL gravity. At long last, in Sec. VI, we present our conclusions.

\section{INTRODUCTION OF QNMS}

QNMs have significant role in many aspects, for example, these modes help us to find hidden information of BHs. By evaluating the QNMs, one can easily find out more accurate information of mass, charge, and other parameters of BHs. The oscillation frequency is determined by real part of frequencies while the imaginary part find out the decay rate at which each mode is damped. Moreover, the QNMs frequencies of AdS BHs have an interpretation in the dual conformal field theory.

In this section, we evaluate the complex frequencies related with QNM of BH by discussing the oscillations of a scalar field. The equation for perturbations is given by [28]-[30]

$$
\frac{1}{|g|} \partial_{u}\left(\sqrt{|g|} \partial^{u} R\right)=0
$$

where $g$ is determinant of the metric. This general perturbation equation is known as Klein-Gordon wave equation. The explicit radial form of this master equation can be written as

$$
f(r)^{2} R^{\prime \prime}(r)+f^{\prime}(r) f(r) R^{\prime}(r)+U R(r)=0,
$$

where prime ( ${ }^{\prime}$ ) denotes derivative w.r.t coordinate $r$ and $U=\omega^{2}-V$.

The tortoise coordinate $\left(r_{*}\right)$ can be defined in differential form as

$$
d r_{*}=\frac{d r}{f(r)},
$$

we can get the Schrödinger-like equation as follows

$$
\frac{d^{2} R}{d r_{*}}+\left(\omega^{2}-V\right) R=0
$$

where the effective potential $V$ can be written as [31]

$$
V=f\left(r_{0}\right)\left(\frac{3 r_{0} f^{\prime}\left(r_{0}\right)}{2 r_{0}^{2}}+\frac{3 f\left(r_{0}\right)}{4 r_{0}^{2}}+\frac{l(l+2)}{r_{0}^{2}}\right) .
$$

The general form of complex frequencies is given by $\omega=\omega_{r}-i \omega_{i}$, which leads to real and imaginary parts. However, QNMs must satisfy the following boundary conditions:

$$
R=\left\{\begin{array}{cc}
e^{-i \omega r_{*},} & r \rightarrow r_{\dot{+}}\left(r_{*} \rightarrow-\infty\right) \\
e^{i \omega r_{*},} & r \rightarrow \infty\left(r_{*} \rightarrow \infty\right)
\end{array},\right.
$$

these boundary conditions are associated with $1 D$ Shrödinger like wave equation. Furthermore, in eikonal regime $l \ggg 1$, for quasinormal resonance frequencies of scalar field perturbation, the WKB resonance condition is defined as [33]

$$
\frac{\eta_{0}}{\sqrt{2 \eta_{0}^{(2)}}}=-i\left(n+\frac{1}{2}\right)+O\left(\frac{1}{l}\right)
$$


where $n$ is overtone number

$$
\begin{gathered}
\eta_{0}=\omega^{2}-V\left(r=r_{0}\right), \\
\eta_{0}^{(2)}=\frac{d^{2} \eta\left[r\left(r_{*}\right)\right]}{d r_{*}^{2}} .
\end{gathered}
$$

Analytical solution of Eq. (7) with real and imaginary parts regarding quasinormal resonance leads to following

$$
\frac{\omega^{2}-\frac{k_{l} f\left(r_{0}\right)}{r_{0}^{2}}}{f\left(r_{0}\right) \sqrt{2 V_{0}^{\prime \prime}}}=-i\left(n+\frac{1}{2}\right)
$$

where $l$ represents the spherical harmonic index and known as orbital angular momentum. The real part of quasinormal resonance modes for $\mathrm{BH}$ can be obtained as

$$
\omega_{R}=\frac{\sqrt{k_{l} f\left(r_{0}\right)}}{r_{0}}
$$

the real and imaginary parts of quasinormal resonance modes can be obtained by using $\omega=\omega_{R}-i \omega_{I}$, whşch yields as

$$
2 \omega_{I} \omega_{R}=\left(n+\frac{1}{2}\right) f\left(r_{0}\right) \sqrt{2 V_{0}^{\prime \prime}}
$$

From Eqs.(11) and (12), one can write the imaginary part of quasinormal resonance frequencies as follows

$$
\omega_{I}=\frac{(2 n+1) r_{0} \sqrt{f\left(r_{0}\right) V_{0}^{\prime \prime}}}{4 \sqrt{k_{l}}}
$$

\section{HAYWARD BH IN EINSTEIN GB GRAVITY}

We start by taking an exact spherically symmetric regular $\mathrm{BH}$ with minimal coupling and nonlinear electrodynamics $[34,35]$. In this session, we will focus on QNMs of Hayward-like BH with regular center in 5D EGB gravity. This BH solution has the following static and spherically symmetric metric [36]:

$$
d s^{2}=-f(r) d t^{2}+(f(r))^{-1} d r^{2}+r^{2} d \Omega_{3}^{2},
$$

where $d \Omega_{3}^{2}=d \theta^{2}+\sin ^{2} \theta\left(d \phi^{2}+\sin ^{2} \phi d \psi^{2}\right)$ is the metric in $3 \mathrm{D}$ hypersurface having volume $V_{3}$. The metric function $f(r)$ is given by

$$
f(r)=1+\frac{r^{2}}{4 \alpha}\left(1+\sqrt{1+\frac{8 \alpha m}{e^{4}+r^{4}}}\right) .
$$

Here, $m$ is a constant of integration associated with the Arnowitt-Deser-Misner (ADM) mass of the BH. From Eqs. (5) and (15), the effective potential of Hayward BH in EGB gravity can be obtained as follow

$$
V_{0}=\frac{1}{4 r_{0}^{2}}\left[\left(\frac{\xi r_{0}^{2}}{4 \alpha}+1\right)\left(4 k_{l}-\frac{24 m r_{0}^{5}}{(\xi-1)\left(r_{0}^{4}+e^{4}\right)^{2}}+\frac{3 \xi r_{0}^{2}}{4 \alpha}+\frac{3 \xi r_{0}}{\alpha}+3\right)\right]
$$

where in this equation

$$
\xi=1+\sqrt{1+\frac{8 \alpha m}{r_{0}^{4}+e^{4}}}
$$

and $k_{l}=l(l+2)$. Now by using the definition of quasinormal resonance mode discussed in previous section, Eqs. (11) and (15) help us to evaluate the real part of the quasinormal frequencies:

$$
\omega_{R}=\frac{1}{r_{0}}\left[k l\left(1+\frac{r_{0}^{2}}{4 \alpha}\left(1+\sqrt{1+\frac{8 \alpha m}{e^{4}+r_{0}^{4}}}\right)\right)\right]^{\frac{1}{2}} .
$$


Substituting the values of Eqs. (15) and (16) in (13), the imaginary part of the quasinormal frequencies can be obtained as

$$
\omega_{I}=\frac{(2 n+1) r_{0}\left[\left(1+\frac{r_{0}^{2}}{4 \alpha}\left(1+\sqrt{1+\frac{8 \alpha m}{e^{4}+r_{0}^{4}}}\right)\right) V_{0}^{\prime \prime}\right]^{\frac{1}{2}}}{4 \sqrt{k_{l}}} .
$$

where

$$
\begin{aligned}
V_{0}^{\prime \prime} & =\frac{48 \eta m r_{0}^{5}\left(11\left(e^{4}+6 \alpha m\right)+5 r_{0}^{4}\right)}{\left(e^{4}+r_{0}^{4}\right)^{2}\left(e^{4}+8 \alpha m+r_{0}^{4}\right)^{2}}+\left(1 2 m r _ { 0 } ^ { 8 } \left(\eta^{3}\left(e^{4}+r_{0}^{4}\right)+\eta^{2}\left(e^{4}+r_{0}^{4}\right)\right.\right. \\
& -2 \alpha m))\left(\alpha \eta^{3}\left(e^{4}+r_{0}^{4}\right)^{4}\right)^{-1}+\left(9 6 \eta m r _ { 0 } ^ { 9 } \left(2 r_{0}^{4}\left(e^{4}-4 \alpha m\right)-3\left(e^{8}+16 \alpha e^{4}\right.\right.\right. \\
& \left.\left.\left.\times m+80 \alpha^{2} m^{2}\right)+5 r_{0}^{8}\right)\right)\left(\left(e^{4}+r_{0}^{4}\right)^{3}\left(e^{4}+8 \alpha m+r_{0}^{4}\right)^{3}\right)^{-1}+\frac{3(\eta+1)}{2 \alpha r_{0}^{3}} \\
& +\frac{16 \eta\left(2 l^{2}+4 l+3\right) m r_{0}^{6}\left(e^{4}+6 \alpha m+r_{0}^{4}\right)}{\left(e^{4}+r_{0}^{4}\right)^{2}\left(e^{4}+8 \alpha m+r_{0}^{4}\right)^{2}}-\frac{768 \eta m r_{0}^{13}\left(e^{4}+5 \alpha m+r_{0}^{4}\right)}{\left(e^{4}+r_{0}^{4}\right)^{3}\left(e^{4}+8 \alpha m+r_{0}^{4}\right)^{3}} \\
& +\frac{3\left(4 l^{2}+8 l+3\right)}{2 r_{0}^{4}}-\frac{48 m r_{0}}{\eta\left(e^{4}+r_{0}^{4}\right)^{2}}-\frac{6\left(2 l^{2}+4 l+3\right) m r_{0}^{2}}{\eta\left(e^{4}+r_{0}^{4}\right)^{2}}+\frac{3\left(\eta^{2}+2 \eta+1\right)}{32 \alpha^{2}} \\
& -\frac{60(\eta+1) m r_{0}^{3}}{\alpha \eta\left(e^{4}+r_{0}^{4}\right)^{2}}+\frac{12}{\alpha\left(e^{4}+r_{0}^{4}\right)^{4}\left(e^{4}+8 \alpha m+r_{0}^{4}\right)^{3}}\left(1 6 r _ { 0 } ^ { 8 } \left(e^{8}(\eta+1)+\alpha e^{4}\right.\right. \\
& \left.\times(5 \eta+14) m+r_{0}^{4}\left(2 e^{4}(\eta+1)+\alpha(5 \eta+14) m\right)+48 \alpha^{2} m^{2}+(\eta+1) r_{0}^{8}\right) \\
& -4 r_{0}^{4}\left(e^{12}(\eta+1)+1536 \alpha^{3} m^{3}-3(\eta+1) r_{0}^{12}+20 \alpha e^{8}(\eta+2) m+8 \alpha^{2} e^{4}\right. \\
& \times(15 \eta+56) m^{2}-r_{0}^{8}\left(5 e^{4}(\eta+1)+16 \alpha m\right)+r_{0}^{4}\left(-e^{8}(\eta+1)+4 \alpha e^{4}(5 \eta\right. \\
& \left.\left.+6) m+8 \alpha^{2}(15 \eta+32) m^{2}\right)\right)+\left(1 7 \left(e^{8}(\eta+1)+2 \alpha e^{4}(3 \eta+8) m+64 \alpha^{2}\right.\right. \\
& \left.\left.\times m^{2}\right)+9(\eta+1) r_{0}^{8}+2 r_{0}^{4}\left(13 e^{4}(\eta+1)+\alpha(51 \eta+104) m\right)\right)
\end{aligned}
$$

and

$$
\eta=\sqrt{\frac{e^{4}+8 \alpha m+r_{0}^{4}}{e^{4}+r_{0}^{4}}}
$$

We can get the effective potential's maximum value at the following point

$$
r_{0}=\frac{1}{\sqrt{3}}\left(m-2 \alpha-\frac{\left(\lambda+\sqrt{\lambda^{2}+4\left(3 e^{4}-(m-2 \alpha)\right)^{3}}\right)^{2}+2^{2 / 3}\left(3 e^{4}-(m-2 \alpha)^{2}\right)}{\sqrt[3]{2}\left(\lambda+\sqrt{\lambda^{2}+4\left(3 e^{4}-(m-2 \alpha)\right)^{3}}\right)}\right)^{\frac{1}{2}}
$$

where

$$
\lambda=-18 e^{4}(4 \alpha+m)+12 \alpha^{2} m+2(m-2 \alpha)^{2} .
$$

\section{A. Wavelike of Hayward BH in EGB gravity}

We rewrite the wavelike Eq. (7) without imposing the stationary limit $R \sim e^{-i \omega t}$.

$$
\frac{\partial^{2} R}{\partial t^{2}}-\frac{\partial^{2} R}{\partial r_{*}^{2}}+V R=0
$$

Following this, here we will provide a complete evolution graph (Figure 7) of the Hayward BH in EGB gravity from a single master equation. 


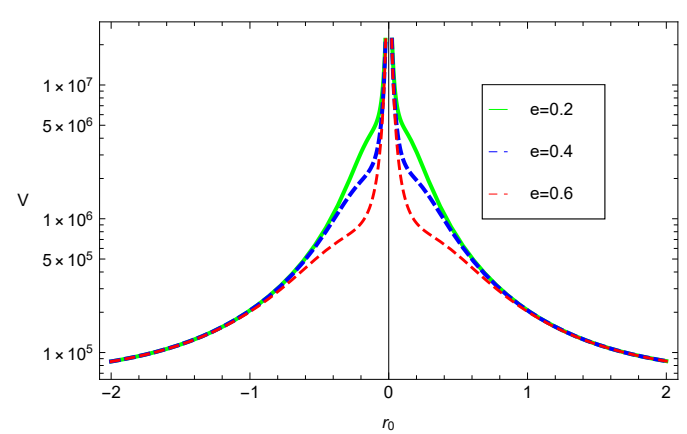

FIG. 1: Effective potential $V$ vs $r_{0}$ for $5 \mathrm{D}$ EGB-Hayward BH. We set $m=20, \alpha=$ 0.08 and $l=100$.

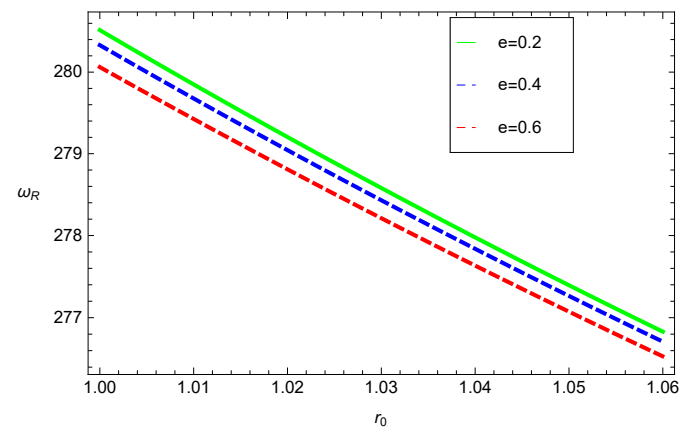

FIG. 3: Real part of quasinormal frequencies $\omega_{R}$ of $5 \mathrm{D}$ EGB-Hayward BH vs $r_{0}$. We set $l=100, \alpha=0.8$ and $m=0.5$.

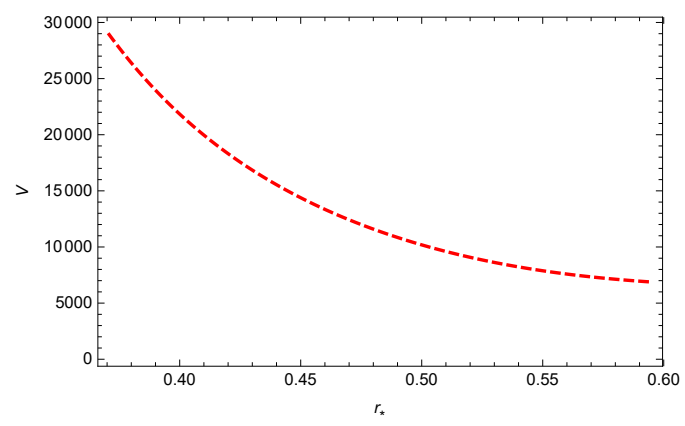

FIG. 5: Plot of effective potential $V$ of $5 \mathrm{D}$ EGB-Hayward BH vs $r_{*}$. We set $\alpha=0.8$, $l=100 e=0.8 m=0.5$.

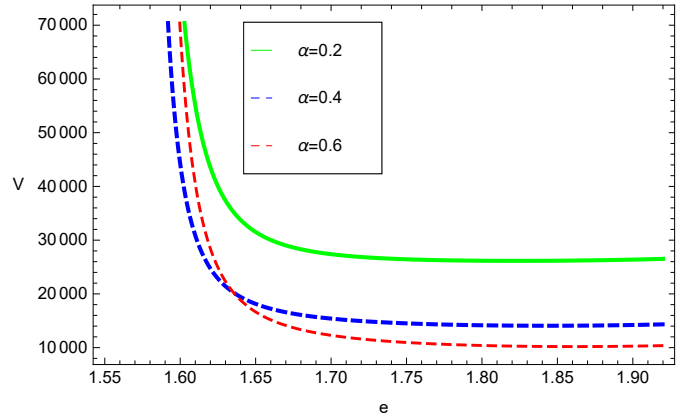

FIG. 2: Effective potential $V$ vs $e$ for 5D EGB-Hayward BH. We set $m=10$ and $l=100$.

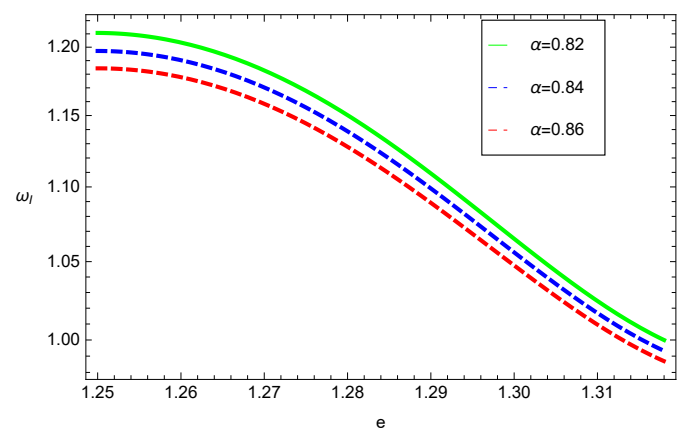

FIG. 4: Imaginary part of the quasinormal frequencies $\omega_{I}$ vs $e$. We set $l=100$ and $m=0.5$.

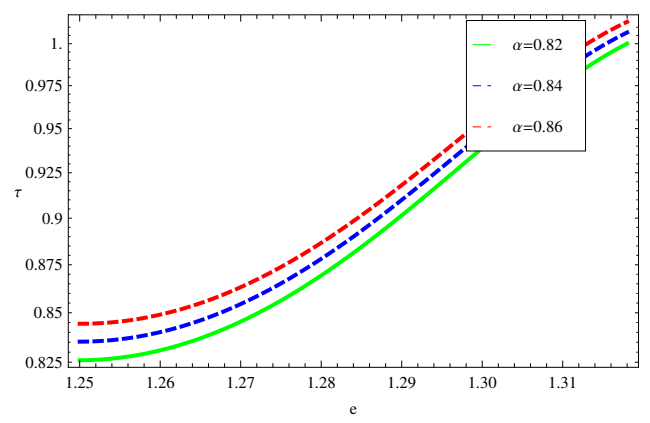

FIG. 6: 2. The relaxation rate for $5 \mathrm{D}$ EGB-Hayward $\mathrm{BH}$ against $e$ with $\mathrm{M}=1$.

\begin{tabular}{|c|c|c|}
\hline \hline Harmonic index number & Overtone & QNMs frequencies using first order WKB method \\
\hline \hline \multirow{3}{*}{$\mathrm{l}=90$} & $n=0$ & $261.786-1.205 i$ \\
& $n=1$ & $265.319-3.615 i$ \\
& $n=2$ & $266.319-6.025 i$ \\
\hline \multirow{4}{*}{$\mathrm{l}=95$} & $n=0$ & $275.396-1.205 i$ \\
& $n=1$ & $279.061-3.614 i$ \\
& $n=2$ & $280.061-6.023 i$ \\
\hline \multirow{3}{*}{$\mathrm{l}=100$} & $n=0$ & $289.006-1.204 i$ \\
& $n=1$ & $292.802-3.612 i$ \\
& $n=2$ & $293.802-6.019 i$ \\
\hline
\end{tabular}

TABLE I: QNMs frequencies using first order WKB method of Hayward BH in Einstein GB gravity. We set $\alpha=0.8$ and $m=0.5$. 


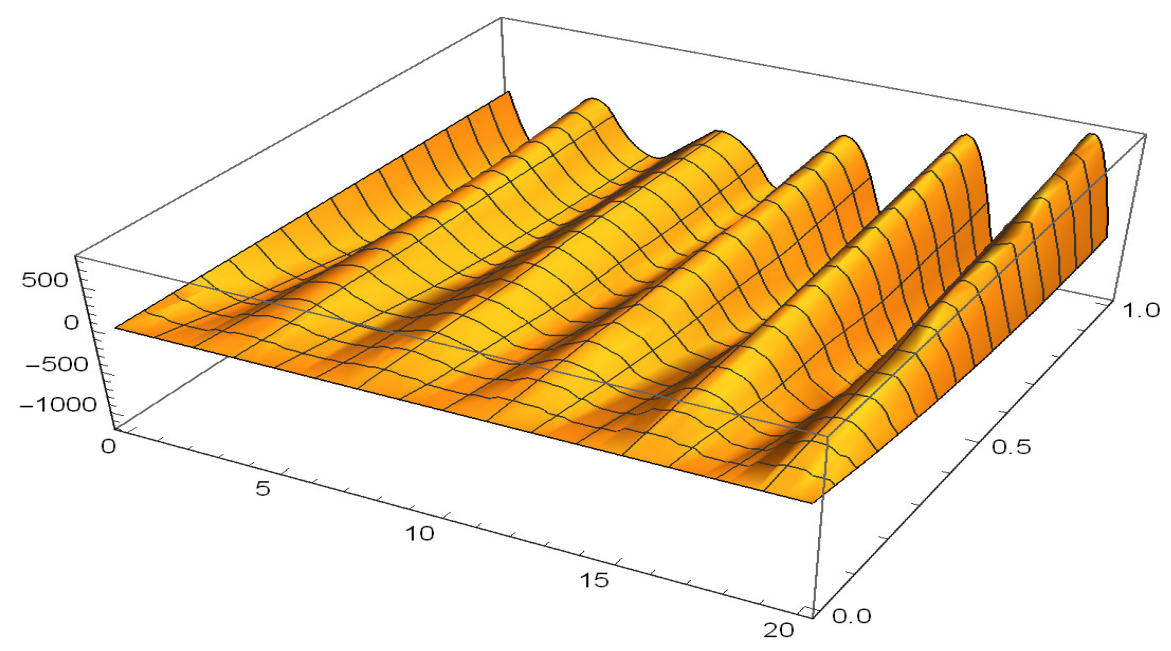

FIG. 7: The plot $R$ of 5D EGB-Hayward BH. We set $\alpha=0.8, l=100 e=0.8 m=0.5$.

In Figure 1 we analyze the impact of magnetic charge $e$ on the effective potential of 5D EGB-Hayward BH. The shape of different trajectories seems alike for increasing values of magnetic charge. The important aspect of trajectories is that the extreme point of effective potential remains same and trajectories decreases with increasing values of magnetic charge of $\mathrm{BH}$ which is similar with RN-BH [43]. Figure 2 demonstrates the impact of coupling constant $\alpha$ on the effective potential of $\mathrm{BH}$. The extreme point of the effective potential decreases with increasing values of coupling constant $\alpha$ for fixed mass. Figure $\mathbf{3}$ and Figure 4 demonstrate the real and the imaginary parts of the QNM frequencies of $\mathrm{BH}$. We observe that real and the imaginary components are not linear functions of $r_{0}$ similar to RN-AdS [43]. As $e$ and $\alpha$ increases the real and imaginary components decreases. The AdS/CFT correspondence suggest that for large $e$, quasinormal ringing becomes slower to settle down to thermal equilibrium and also, the frequency of the oscillation becomes smaller. Our results are very much consistent with literature work [42]. Figure 5 demonstrates the behavior of the effective potential versus the tortoise coordinater. The effective potential decreases with increasing values of tortoise coordinate $r_{*}$ with fixed electric charge, coupling constant and mass of 5D BH in Einstein GB gravity. Figure 6 shows the relaxation time of the 5D EGB-Hayward BH in terms of the reduced charge of the $\mathrm{BH}$. The relaxation rate is increasing function of charge of the $\mathrm{BH}$. We also observed that the relaxation time increases with increasing values of parameter $\alpha$.

In Figure $\mathbf{7}$ one can get a time-domain 3-dimensional profile of the perturbation. When looking at Figure $\mathbf{7}$ a natural question is to which value of the quasinormal frequency is calculated, we solve numerically through finite difference method. For finite values of $-r *$ and $r *$ and smaller $\alpha$ and $m$ we obtain the stable region of hyperbolic equation.

\section{HAYWARD BH IN ADS SPACETIME}

In this session, we consider Hayward-AdS BH by introducing a new order variable, the potential conjugate of magnetic charge $Q_{m}$ in view of the non-linearly coupled electromagnetic field. The static spherically symmetric solution is given by $[37,38]$

$$
d s^{2}=-f(r) d t^{2}+(f(r))^{-1} d r^{2}+r^{2} d \Omega^{2},
$$

where $d \Omega^{2}=d \theta^{2}+\sin ^{2} \theta d \phi^{2}$. In AdS background the metric function $f(r)$ has the form

$$
f(r)=\frac{r^{2}}{l^{2}}+1-\frac{2 M_{S}}{r}-\frac{2 \alpha^{-1} g^{3} r^{u-1}}{g^{u}+r^{u}}
$$

where $M_{S}$ represents the Schwarzschild mass and $g$ denotes the integration constant which is related to magnetic charge and it is defined as

$$
Q_{m}=\frac{g^{2}}{\sqrt{2 \alpha}}
$$


By taking cosmological constant as thermodynamical pressure $P=-\frac{\Lambda}{8 \pi}=\frac{3}{8 \pi l^{2}}$, the Hayward BH solution in AdS for $u=3$, leads to

$$
f(r)=\frac{8}{3} \pi \operatorname{Pr}^{2}+1-\frac{2 M_{S}}{r}-\frac{2^{7 / 4} r^{2} Q_{m}^{3 / 2}}{\sqrt{\alpha}\left(\left(\sqrt{2 \alpha} Q_{m}\right)^{3 / 2}+r^{3}\right)} .
$$

The effective potential for 4D Hayward $\mathrm{BH}$ in AdS spacetime is given by [41]

$$
V=f\left(r_{0}\right)\left(\frac{f^{\prime}\left(r_{0}\right)}{r_{0}}+\frac{l(l+1)}{r_{0}^{2}}\right)
$$

From Eqs.(23) and (24), the effective potential of the considered BH takes the form

$$
\begin{aligned}
V & =\left(\frac{8}{3} \pi P r_{0}^{2}+1-\frac{22^{3 / 4} r_{0}^{2} Q_{m}^{3 / 2}}{\sqrt{\alpha}\left(2^{3 / 4}\left(\sqrt{\alpha} Q_{m}\right)^{3 / 2}+r_{0}^{3}\right)}-\frac{2 M_{S}}{r_{0}}\right) \frac{1}{r_{0}}\left(\frac{l(l+1)}{r_{0}}\right. \\
& +\frac{2 M_{S}}{r_{0}^{2}}+\frac{16}{3} \pi P r_{0}-\frac{42^{3 / 4} r_{0} Q_{m}^{3 / 2}}{\sqrt{\alpha}\left(2^{3 / 4}\left(\sqrt{\alpha} Q_{m}\right)^{3 / 2}+r_{0}^{3}\right)}+\left(62^{3 / 4} r_{0}^{4} Q_{m}^{3 / 2}\right) \\
& \left.\times\left(\sqrt{\alpha}\left(2^{3 / 4}\left(\sqrt{\alpha} Q_{m}\right)^{3 / 2}+r_{0}^{3}\right)^{2}\right)^{-1}\right) .
\end{aligned}
$$

The real part of QNM frequency $\omega_{R}$ for $4 \mathrm{D}$ Hayward BH in AdS spacetime turns out to be

$$
\omega_{R}=\frac{1}{r_{0}}\left[l(l+1)\left(\frac{8}{3} \pi \operatorname{Pr}_{0}^{2}+1-\frac{22^{3 / 4} r_{0}^{2} Q_{m}^{3 / 2}}{\sqrt{\alpha}\left(2^{3 / 4}\left(\sqrt{\alpha} Q_{m}\right)^{3 / 2}+r_{0}^{3}\right)}-\frac{2 M_{S}}{r_{0}}\right)\right]^{\frac{1}{2}} .
$$

Moreover, one can find the imaginary part of QNM frequency $\omega_{I}$ as follows

$$
\omega_{I}=\frac{r_{0} \sqrt{V_{0}^{\prime \prime}}}{2 \sqrt{2 k_{l}}}\left[\frac{8}{3} \pi \operatorname{Pr}_{0}^{2}+1-\frac{2 M_{S}}{r_{0}}-\frac{2^{7 / 4} r_{0}^{2} Q_{m}^{3 / 2}}{\sqrt{\alpha}\left(\left(\sqrt{2 \alpha} Q_{m}\right)^{3 / 2}+r_{0}^{3}\right)}\right]^{\frac{1}{2}}
$$

where $k_{l}=l(l+1), l$ is angular momentum parameter, $r_{0}$ represents radii of outer event horizon and $V_{0}^{\prime \prime}$ denotes the second order derivative of effective potential of the Hayward BH in AdS Spacetime.

\section{A. Wavelike of Hayward BH in AdS spacetime}

We rewrite the wavelike Eq.(7) without imposing the stationary limit $R \sim e^{-i \omega t}$.

$$
\frac{\partial^{2} R}{\partial t^{2}}+V R=\frac{\partial^{2} R}{\partial r_{*}^{2}}
$$

Here $V=f\left(r_{0}\right)\left(\frac{f^{\prime}\left(r_{0}\right)}{r_{0}}+\frac{l(l+1)}{r_{0}^{2}}\right)$ and $f\left(r_{0}\right)$ is the metric function od Hayward BH in AdS spacetime. Following this, we will provide a complete evolution graph of Hayward BH in AdS spacetime from a single master equation.

Figures 8, 9 demonstrate the behavior of effective potential with fixed/constant boundaries (at the infinity and event horizon), it reaches to maximum value at some intermediate values of $r_{0}$. The behavior of graph becomes monotonically decreasing after reaching to maximum value and vise versa, which is a different from the 5D BHs [46]. The impact of magnetic charge on the effective potential is important for larger values of $r_{0}$ while the influence of mass is significant for smaller $r_{0}$. Figure 10, 11 show the impact of charge and mass on the real part of quasinormal frequencies $\omega_{R}$ of Hayward AdS BH. For increasing values of charge and mass trajectories of $\omega_{R}$ decreases. As magnetic charge $Q_{m}$ increases, a linear relation with $r_{0}$ no longer holds. We observe that as magnetic charge increases, $\omega_{R}$ decreases. According to AdS spacetime correspondence, it means for large $Q_{m}$, it decreases for quasinormal to settle down. Figure 12, 13 show the impact of charge and angular momentum parameter on the imaginary part of quasinormal frequencies $\omega_{R}$ of Hayward AdS BH. As the magnetic charge and angular momentum parameter increases the trajectories of imaginary part of quasinormal frequencies $\omega_{R}$ of Hayward AdS BH decreases. Now we can make a sense in Figures 14, 15, where we can analyze that, for the $4 \mathrm{D}$ self-possessed system, the second derivative of 


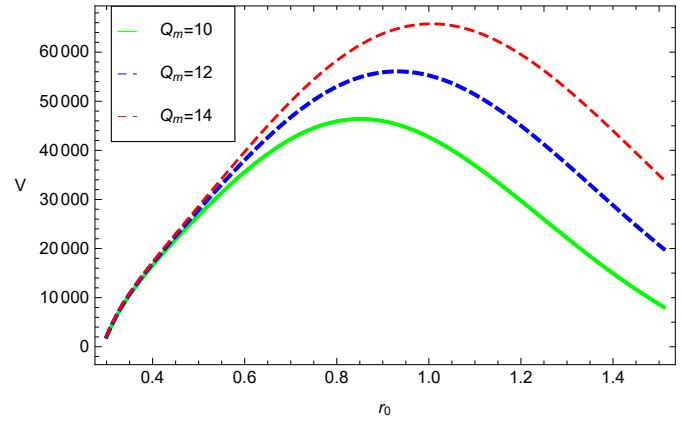

FIG. 8: The behavior of effective potential $V$ of Hayward AdS BH in terms of $r_{0}$. We set $\alpha=0.8, P=0.5, M_{S}=0.5$ and $l=2$.

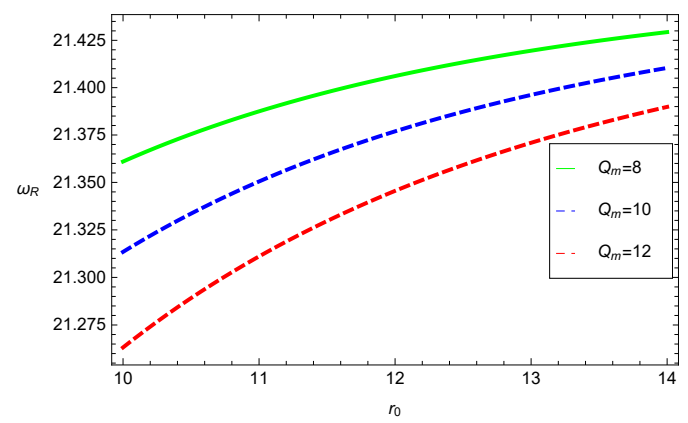

FIG. 10: The real part of quasinormal frequencies $\omega_{R}$ of Hayward AdS BH vs $r_{0}$. We set $\alpha=0.8, P=0.5, M_{S}=0.5$ and $l=2$.

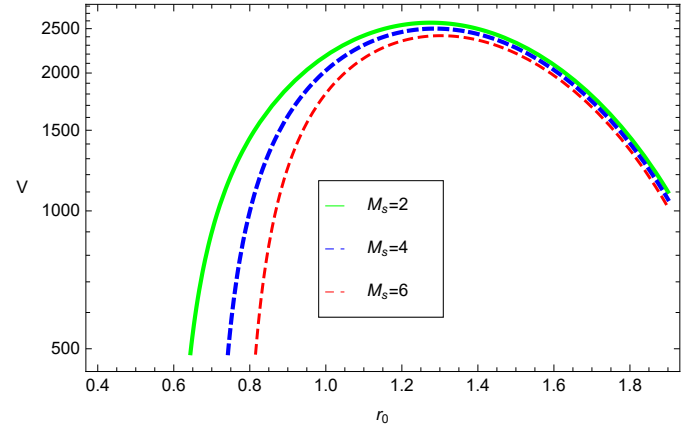

FIG. 9: The behavior of effective potential $V$ of Hayward AdS BH in terms of $r_{0}$. We set $\alpha=0.08, P=0.5, Q_{m}=12$ and $l=2$.

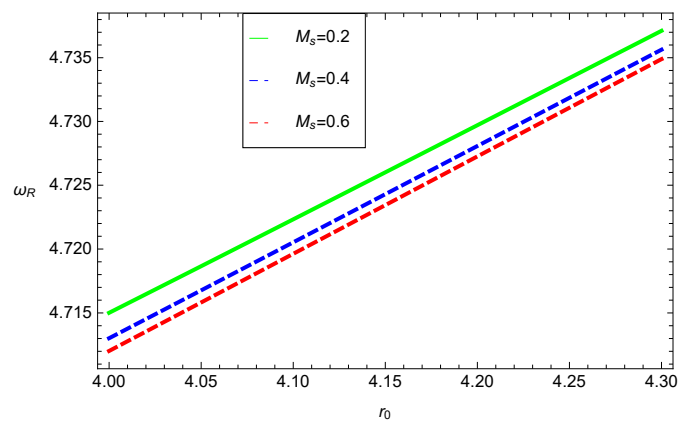

FIG. 11: The real part of quasinormal frequencies $\omega_{R}$ of Hayward AdS BH vs $r_{0}$. We set $\alpha=2, P=0.5, Q_{m}=12$ and $l=2$.

the $V_{0}$ increases for increasing values of $Q_{m}$, it reaches to its maximum value then it decreases for higher values of $r_{0}$. While for increasing values of $M_{s}$ the trajectories decreases and show the alternating increasing and decreasing behavior against $Q_{m}$. These results are very close to Zhang, M. et al. paper [43]. Figure 16 shows the relaxation time of the 5D EGB-Hayward $\mathrm{BH}$ in terms of the reduced charge of the $\mathrm{BH}$. The relaxation rate decreases and then increases for higher values of charge of the $\mathrm{BH}$. We also observed that the relaxation time increases with increasing values of parameter $l$.

In Figure 17 for Hayward BH in AdS spacetime, one can get a time-domain 3-dimensional profile of the perturbation. We find the value of the quasinormal frequencies numerically through finite difference method. For finite values of $-r *$ and $r *$ and smaller $M_{s}$ and $P$ we obtain the stable region of hyperbolic equation.

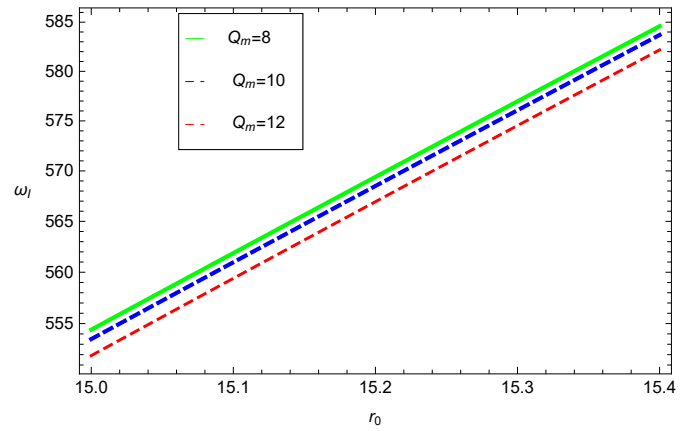

FIG. 12: The imaginary part of quasinormal frequencies $\omega_{I}$ of Hayward AdS BH vs $r_{0}$. We set $\alpha=0.8, P=0.5, M_{S}=0.5$ and $l=2$.

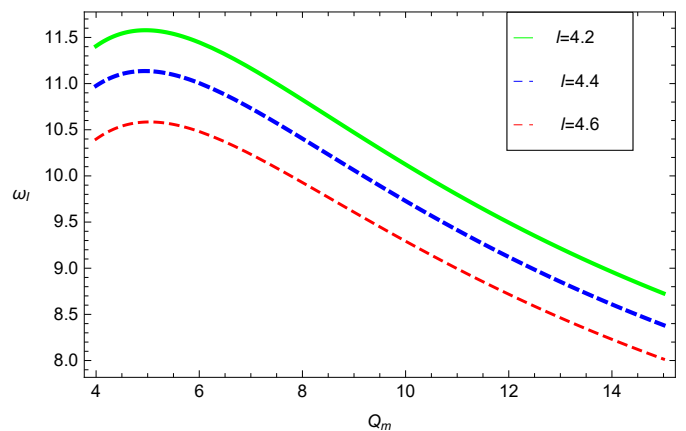

FIG. 13: The imaginary part of quasinormal frequencies $\omega_{I}$ of Hayward AdS BH in terms of $Q_{m}$. We set $\alpha=0.8, P=0.8$, $M_{S}=0.5$. 


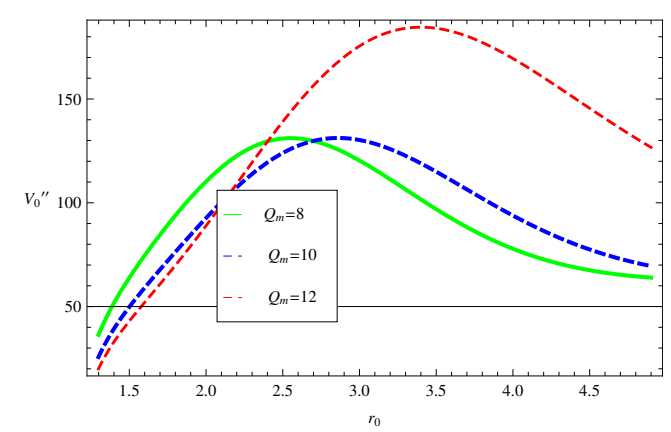

FIG. 14: The behavior of $V_{0}^{\prime \prime}$ in terms of $r_{0}$. We set $\alpha=0.8, P=0.5, M_{S}=0.5$ and $l=2$.

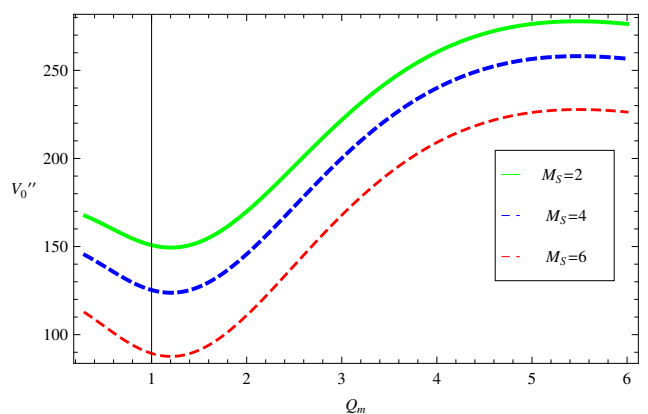

FIG. 15: The behavior of $V_{0}^{\prime \prime}$ in terms of $Q_{m}$. We set $\alpha=0.8, P=0.8$ and $l=2$.

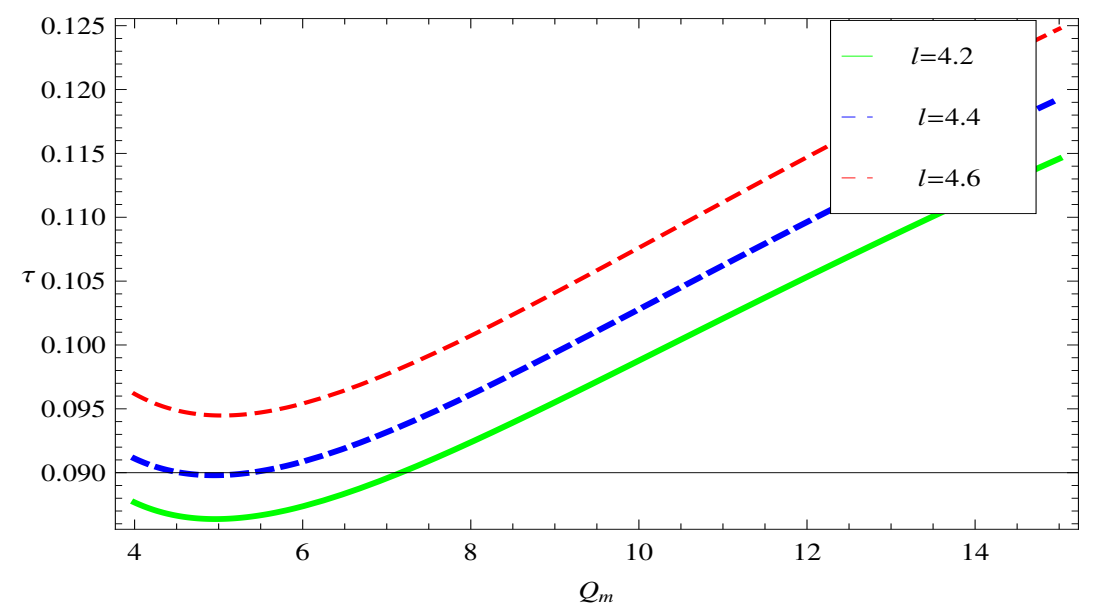

FIG. 16: Plot of relaxation rate for Hayward AdS BH against $Q_{m}$ with $\mathrm{M}=1$.

\section{BH IN 4D EL GRAVITY}

Glavan and Lin recently formulated 4D EGB theory of gravity which is different from the pure Einstein theory [44]. In this session we study QNM to BHs in 4D EL theory with cosmological constant $\Lambda$ (either negative or positive) [39]. The general form of D-dimensional static as well as maximally symmetric metric is given by

$$
d s^{2}=-f(r) d t^{2}+(f(r))^{-1} d r^{2}+r^{2} \gamma_{i j} d x^{i} d x^{j},
$$

\begin{tabular}{|c|c|c|}
\hline \hline Harmonic index number & Overtone & QNMs frequencies using first order WKB method \\
\hline \hline \multirow{3}{*}{$l=3$} & $n=0$ & $5.32383-0.99597 i$ \\
& $n=1$ & $6.32383-2.98793 i$ \\
& $n=2$ & $7.32383-4.97989 i$ \\
\hline \multirow{3}{*}{$l=4$} & $n=0$ & $6.02639-0.67421 i$ \\
& $n=1$ & $7.02639-2.02265 i$ \\
& $n=2$ & $8.02639-3.37109 i$ \\
\hline & $n=0$ & $6.72753-0.29855 i$ \\
& $n=1$ & $7.72753-0.89566 i$ \\
& $n=2$ & $8.72753-1.29278 i$ \\
\hline
\end{tabular}

TABLE II: QNMs frequencies using first order WKB method of Hayward BH in AdS spacetime. We set $\alpha=0.8, P=0.5$, $r_{0}=1.27$ and $M_{S}=0.5$. 


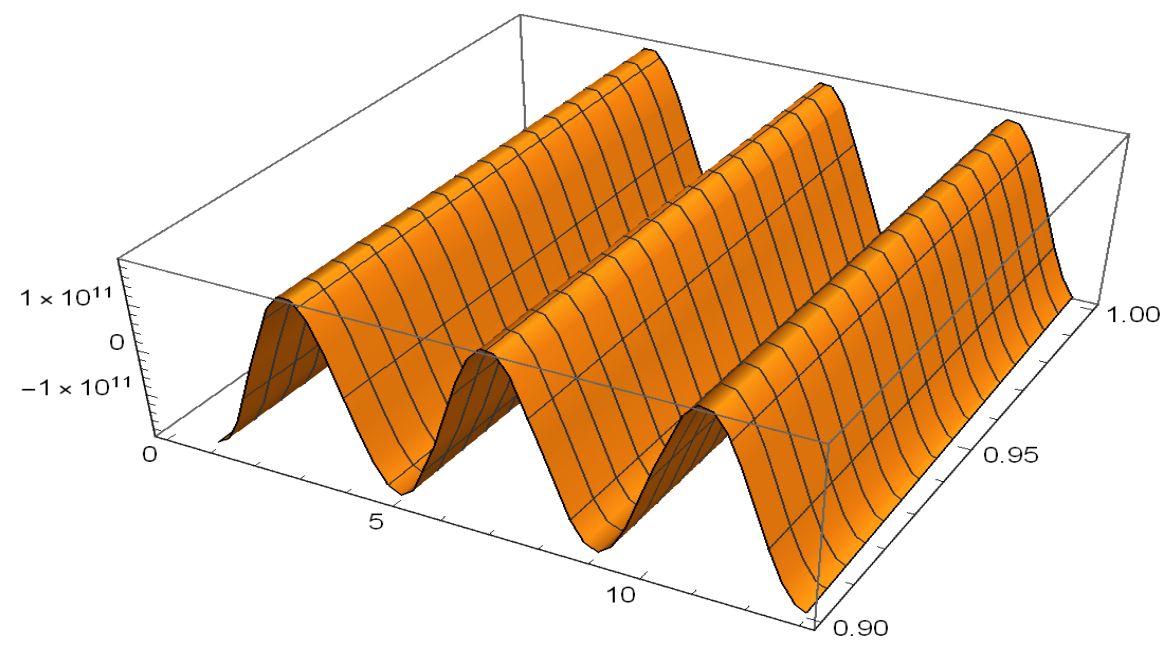

FIG. 17: The plot $R$ for Hayward AdS BH. Set $M=1$.

where $d \Omega_{n}^{2}$ is $(n=D-2)$-dimensional and constant curvature space with $\kappa=1$ represents spherically symmetric BH solution. For $D=4$, we have two branches of $f(r)$ as [40]

$$
f(r)=1-\frac{r^{2}}{2 \tilde{\alpha_{2}}}\left(-1+-\sqrt{1+4 \tilde{\alpha_{2}}\left(\frac{2 M}{r^{3}}-\frac{Q^{2}}{r^{4}}+\frac{\Lambda}{3}\right)}\right) .
$$

The $(+)$ sign branch is perturbative in $\tilde{\alpha_{2}}$ and for $(-) \operatorname{sign} f(r) \rightarrow \infty$ when $\tilde{\alpha_{2}} \rightarrow 0$. From Eqs. (24) and (28), one can the determined the effective potential of $\mathrm{BH}$ as

$$
V=\left(1-\frac{\lambda r_{0}^{2}}{2 \tilde{\alpha_{2}}}\right)\left(\frac{k_{l}}{r_{0}^{2}}-\frac{\lambda}{\tilde{\alpha_{2}}}+\frac{2\left(3 M r_{0}-2 Q^{2}\right)}{(\lambda+1) r_{0}^{4}}\right)
$$

where

$$
\lambda=-1+\left[1+\tilde{\alpha_{2}}\left(\frac{4 \Lambda}{3}-\frac{4\left(Q^{2}-2 M r_{0}\right)}{r_{0}^{4}}\right)\right]^{\frac{1}{2}} .
$$

The real part of QNM frequency $\omega_{R}$ for the considered BH becomes

$$
\omega_{R}=\frac{1}{r_{0}}\left[k_{l}\left(1-\frac{r_{0}^{2}}{2 \tilde{\alpha_{2}}}\left(-1+\sqrt{4 \tilde{\alpha_{2}}\left(1+\frac{\Lambda}{3}+\frac{2 M}{r_{0}^{3}}-\frac{Q^{2}}{r_{0}^{4}}\right)}\right)\right)\right]^{\frac{1}{2}}
$$

The imaginary part of QNM frequency $\omega_{I}$ for this case turns out to be

$$
\omega_{I}=\frac{\sqrt{2 V_{0}^{\prime \prime}} r_{0}\left[1-\frac{r_{0}^{2}}{2 \tilde{\alpha_{2}}}\left(-1+\sqrt{1+4 \tilde{\alpha_{2}}\left(\frac{2 M}{r_{0}^{3}}-\frac{Q^{2}}{r_{0}^{4}}+\frac{\Lambda}{3}\right)}\right)\right]^{\frac{1}{2}}}{4 \sqrt{k_{l}}} .
$$

\section{A. Wavelike of BH in $4 \mathrm{D}$ EL Gravity}

We rewrite the wavelike Eq.(7) without imposing the stationary limit $R \sim e^{-i \omega t}$.

$$
\frac{\partial^{2} R}{\partial t^{2}}=\frac{\partial^{2} R}{\partial r_{*}^{2}}-V R
$$

Here $V=\left(1-\frac{\lambda r_{0}^{2}}{2 \tilde{\alpha_{2}}}\right)\left(\frac{k_{l}}{r_{0}^{2}}-\frac{\lambda}{\tilde{\alpha_{2}}}+\frac{2\left(3 M r_{0}-2 Q^{2}\right)}{(\lambda+1) r_{0}^{4}}\right)$. Using this, we will provide a complete evolution graph of BH in $4 \mathrm{D}$ Einstein-Lovelock gravity from a single master equation. 


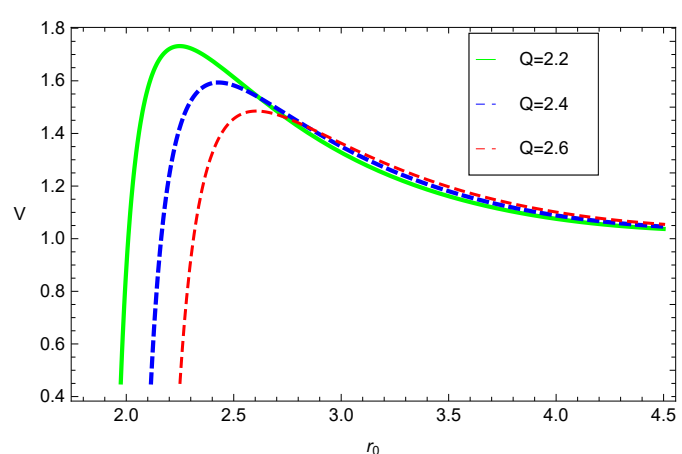

FIG. 18: The effective potential $V$ versus $r_{0}$ for $\mathrm{BH}$ in Einstein-Lovelock gravity. We use $\tilde{\alpha_{2}}=0.8, \Lambda=-0.2, M=0.5$ and $l=2$.

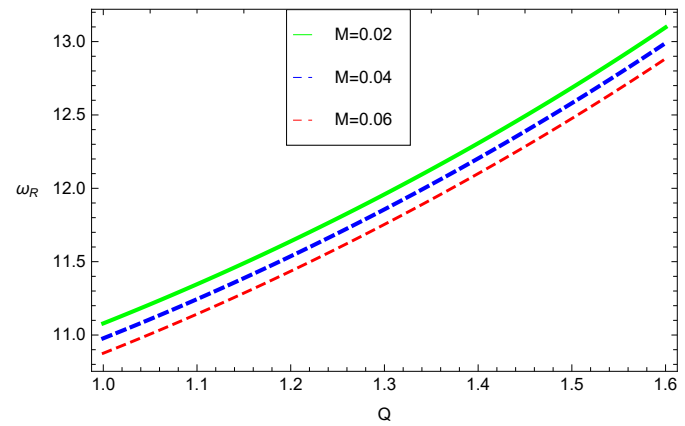

FIG. 20: The variation of $\omega_{R}$ versus $Q$ for $\mathrm{BH}$ in EL gravity. We use $\tilde{\alpha_{2}}=0.8$, $\Lambda=0.05$ and $l=2$.

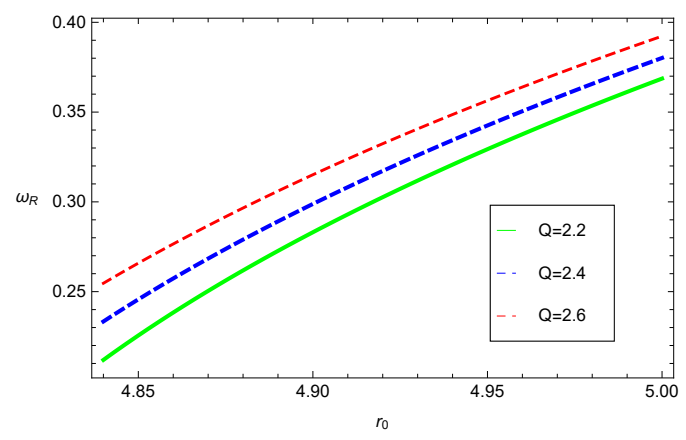

FIG. 19: The variation of $\omega_{R}$ versus $r_{0}$ for $\mathrm{BH}$ in Einstein-Lovelock gravity. We use $\tilde{\alpha_{2}}=0.8, \Lambda=-0.5, M=12$ and $l=2$.

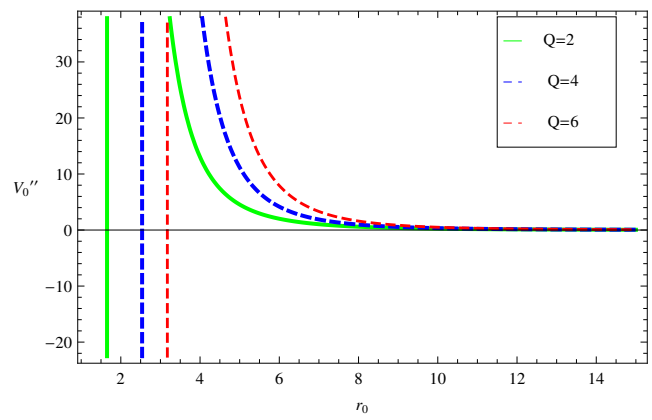

FIG. 21: The variation of $V_{0}^{\prime \prime}$ versus $r_{0}$ for $\mathrm{BH}$ in EL gravity. We use $\tilde{\alpha_{2}}=0.8$, $\Lambda=0.05, M=0.5$ and $l=20$.

From Eq.(29), one can see that $V$ depends upon BH charge, $l$ and $\Lambda$ (negative cosmological constant) and parameter $\tilde{\alpha_{2}}$, we study the dependence of $V$ on these parameters, since the QNMs are decided by the $V$. The behavior of $V$ against $r_{0}$ for some estimation of $Q, \Lambda, \tilde{\alpha_{2}}$ and mass $M$ is plotted in Figure 18 [47]. The plot shows different behaviors of $Q$ on the effective potential as compare to Hayward AdS BH. In Figures 19, 20 the real part of quasinormal frequencies increases with increasing values of electric charge $Q$. When $\Lambda$ is small, the oscillation of massless scalar field increases rapidly and becomes gradually slower as electric charge increases with positive cosmological constant. In Figure 21 for the 4D composed system, the second derivative of $V_{0}$ at the peak point increases with increasing values of parameter $Q$ and the value starts to decrease when $\mathrm{BH}$ reaches to extreme point, the behavior of graph is similar with the RN-BH. In Figure 22 the imaginary part of quasinormal frequencies $\omega_{I}$ increases and then decreases

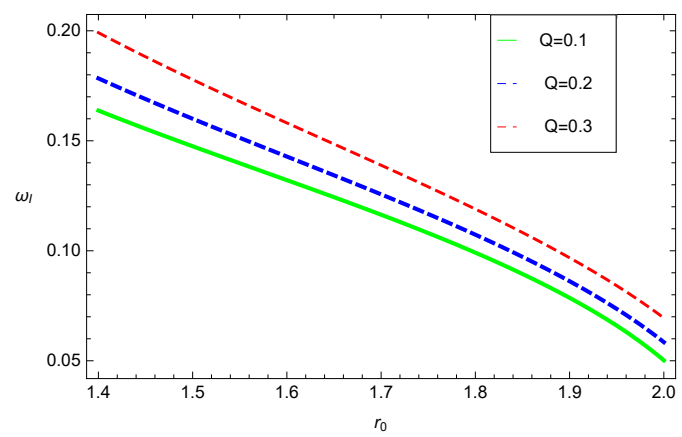

FIG. 22: The variation of $\omega_{I}$ versus $r_{0}$ for $\mathrm{BH}$ in Einstein-Lovelock gravity. We use $\tilde{\alpha_{2}}=0.8, \Lambda=0.5, M=0.5$ and $l=2$.

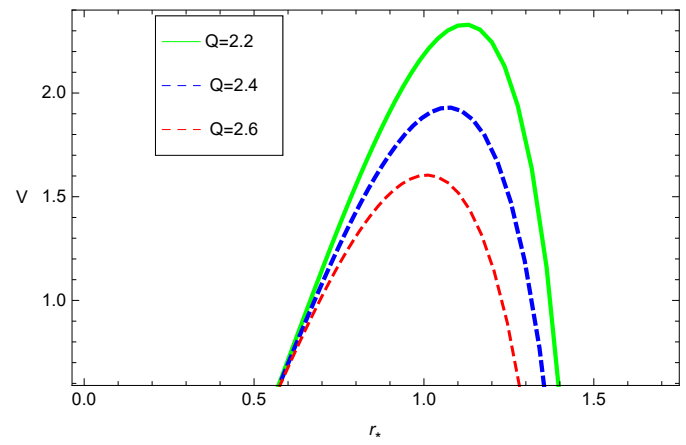

FIG. 23: The variation of $V$ versus $r_{*}$ for $\mathrm{BH}$ in Einstein-Lovelock gravity. We use $\tilde{\alpha_{2}}=0.4, \Lambda=0.5, M=0.5$ and $l=2$. 


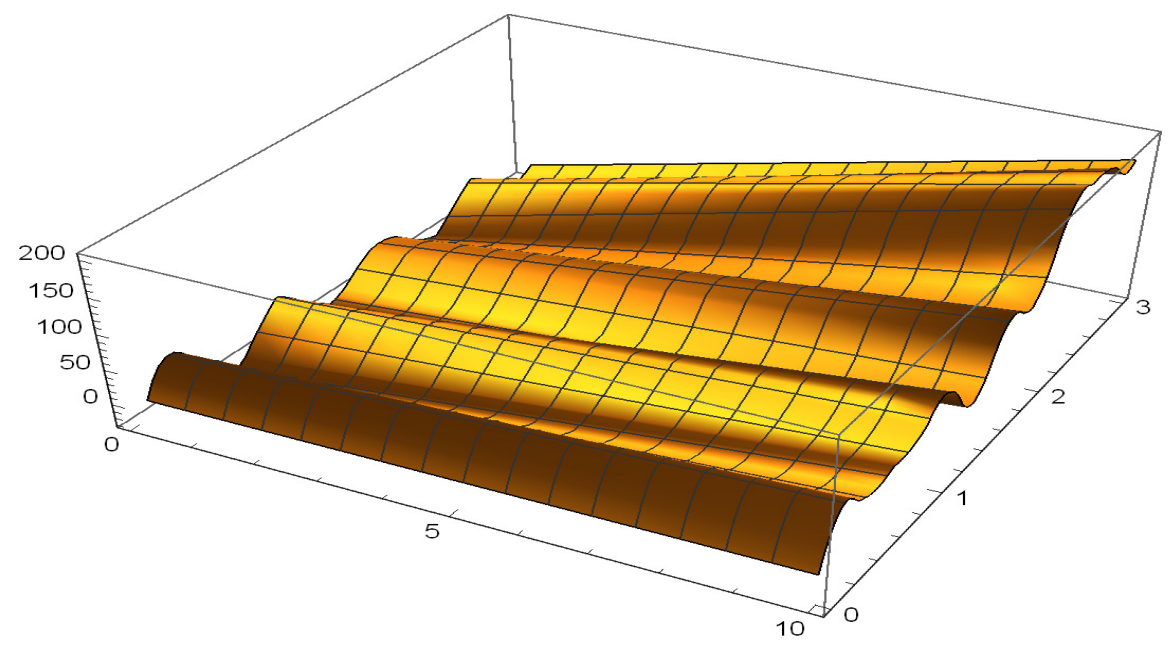

FIG. 24: The plot $R$ for BH in 4D Einstein-Lovelock gravity. We use $\tilde{\alpha_{2}}=0.4, \Lambda=0.5, M=0.5$ and $l=2$.

\begin{tabular}{|c|c|c|}
\hline \hline Harmonic index number & Overtone & QNMs frequencies using first order WKB method \\
\hline \hline \multirow{3}{*}{$l=2$} & $n=0$ & $1.35141-0.16281 i$ \\
& $n=1$ & $2.35141-0.48845 i$ \\
& $n=2$ & $3.35141-0.81409 i$ \\
\hline \multirow{3}{*}{$l=3$} & $n=0$ & $1.70407-0.16164 i$ \\
& $n=1$ & $2.70407-0.48492 i$ \\
& $n=2$ & $3.70407-0.80821 i$ \\
\hline \multirow{3}{*}{$l=4$} & $n=0$ & $2.05445-0.16117 i$ \\
& $n=1$ & $3.05445-0.48351 i$ \\
& $n=2$ & $4.05445-0.48351 i$ \\
\hline
\end{tabular}

TABLE III: QNMs frequencies using first order WKB method of BH in $4 \mathrm{D}$ EL gravity. We set $\tilde{\alpha_{2}}=0.8, \Lambda=0.05, r_{0}=2$ and $Q=1.5$.

for different values of electric charge. For the increasing values of $r_{0}$, the corresponding electric charge increases and $\omega_{I}$ reaches to the maximum value [42]. Figure 23 shows the behavior $V$ with increasing $r_{0}$ for different values of $Q$.

In Figure 24 one can get a time-domain 3-dimensional profile of the perturbation. We find the value of the quasinormal frequencies numerically through finite difference method. For finite values of $-r *$ and $r *$ and smaller cosmological constant and $P$ we obtain the stable region of hyperbolic equation of BH in $4 \mathrm{D}$ EL gravity studied in Eq.(33).

The data presented in in Eqs.(17), (18), (22), (23), (27) and (28) does not accept one to see that the Schwarzschild limit can be reproduced as the mode together with smallest $p$ is rather far from the specific BHs. The correctness of our results is also indicated by "the convergence" obtained these numerical values for the complex frequencies at first order WKB method. This is a important $\omega_{R}>\omega_{I}$ well known condition is satisfied for every value in the table. That is presented in table I, II and in table III.

\section{CONCLUSION}

We have discussed the QNMs for Hayward BH in EGB gravity, in AdS spacetime, and in 4D EL gravity. By employing the WKB technique, we examined the massless scalar QNMs for static regular BHs. The dynamical development of the QNMs are also examined for various estimations of angular momentum and other parameters. We also find out the relaxation rate for the considered BHs and observed that relaxation rate increases with increasing values of charge.

For the case of Hayward BH in EGB gravity, the extreme point of effective potential remains same and trajectories decreases with increasing values of magnetic charge of $\mathrm{BH}$ which is similar with RN-BH [43]. Similar to RN-AdS $\mathrm{BH}$, we observed that real and the imaginary components for Hayward BH in EGB gravity are not linear functions 
of $r_{0}$. The AdS/CFT correspondence suggests that for large $e$, quasinormal ringing becomes slower to settle down to thermal equilibrium and the frequency of the oscillation gets smaller.

For Hayward BH in AdS spacetime, the effective potential becomes monotonically decreasing function after reaching to maximum value which is different from the above case also the impact of magnetic charge on the effective potential is important for larger values of $r_{0}$. For increasing values of charge and mass, the trajectories of $\omega_{R}$ decreases. As magnetic charge $Q_{m}$ increases, linear relation with $r_{0}$ no longer holds. As the magnetic charge and angular momentum parameter increases, the trajectories of imaginary part of quasinormal frequencies $\omega_{R}$ of Hayward AdS BH decreases.

The behavior of $V$ against $r_{0}$ for $\mathrm{BH}$ in $4 \mathrm{D}$ EL gravity is quiet similar to Hayward $\mathrm{BH}$ in AdS spacetime but the influence of charge becomes negligible for higher values of $r_{0}$. The real part of quasinormal frequencies increases with increasing values of electric charge $Q$ while the imaginary part of quasinormal frequencies $\omega_{I}$ increases and then decreases for different values of electric charge.

Since the stationary BH solutions in modified gravity theories have become significant as they can provide a platform to test them through astrophysical observations, in the near future, we plan to generalize our current study to the rotating regular BHs and reveal the effect of the rotation on their QNMs.

\section{Acknowledgements}

Abdul Jawad and Shahid Chaudhary would like to acknowledge HEC, Islamabad, Pakistan for its financial support through the Indigenous 5000 Ph.D. Fellowship Project Phase-II, Batch-V.

[1] B. P. Abbott et al. [LIGO Scientific and Virgo], Phys. Rev. Lett. 116, 061102 (2016).

[2] K. D. Kokkotas and B. G. Schmidt, Living. Rev. Relativ. 2, 2 (1999).

[3] S. W. Hawking and G. F. R. Ellis, Cambridge University Press ( 1973).

[4] A. Övgün, I. Sakalli and J. Saavedra, Chin. Phys. C 42, no.10, 105102 (2018).

[5] A. Övgün and K. Jusufi, Annals Phys. 395, 138-151 (2018)

[6] A. Övgün, I. Sakalli and H. Mutuk, [arXiv:1904.09509 [gr-qc]].

[7] I. Sakalli, K. Jusufi and A. Övgün, Gen. Rel. Grav. 50, no.10, 125 (2018)

[8] P. A. Gonzalez, A. Övgün, J. Saavedra and Y. Vasquez, Gen. Rel. Grav. 50, no.6, 62 (2018)

[9] S. Lepe and J. Saavedra, Phys. Lett. B 617, 174-181 (2005)

[10] J. Crisostomo, S. Lepe and J. Saavedra, Class. Quant. Grav. 21, 2801-2810 (2004)

[11] R. Becar, S. Lepe and J. Saavedra, Phys. Rev. D 75, 084021 (2007)

[12] A. Aragon, R. Becar, P. A. Gonzalez and Y. Vasquez, Eur. Phys. J. C 80, no.8, 773 (2020).

[13] I. Sakalli, Eur. Phys. J. C 75, no.4, 144 (2015).

[14] K. Jusufi, I. Sakalli and A. Övgün, Gen. Rel. Grav. 50, no.1, 10 (2018).

[15] A. Aragón, P. A. González, E. Papantonopoulos and Y. Vásquez, JHEP 08, 120 (2020).

[16] J. Bardeen, presented at GR5, Tiflis, U.S.S.R., and published in the conference proceedings in the U.S.S.R. (1968).

[17] E. Ayon-Beato and A. Garcia, Phys. Lett. B 493, 149 (2000).

[18] S. A. Hayward, Phys. Rev. Lett. 96, 031103 (2006).

[19] K. Lin, J. Li and S. Yang, Int. J. Theor. Phys. 52, 3771 (2013).

[20] M. Halilsoy, A. Ovgun and S. H. Mazharimousavi, Eur. Phys. J. C 74, 2796 (2014).

[21] M. Sharif and S. Mumtaz, Adv. High Eng. Phys. 2016, 2868750 (2016).

[22] I. Sakalli, A. Ovgun and S. F. Mirekhtiary, Int. J. Geom. Meth. Mod. Phys. 11, no.08, 1450074 (2014).

[23] S. S. Zhao and Y. Xie, Eur. Phys. J. C 77, 272 (2017).

[24] B. Gwak, Eur. Phys. J. C 77, 482 (2017).

[25] R. Konoplya, Phys. Rev. D 71, 024038 (2005).

[26] S. K. Chakrabarti, Gen. Relat. Grav. 39567 (2007).

[27] B. F. Schutz and C. M. Will, The Astro. phys. 291, L33-L36 (1985).

[28] R. G. Cai et al., Phy. Rev. D 91, 0224032 (2015).

[29] D. Kubiznak, R. B. Mann, and M. Teo, Class. Quant. Grav. 34 (6), 063001 (2017).

[30] R. A. Konoplya, Phys. Rev. D 66, 084007 (2002).

[31] J. P. M. Graca, I. S. Godonou and V. B. Bezerra, Modrn. Phys. D 261750113 (2017).

[32] G. T. Horowitz and V. E. Hubeny, Phys. Rev. D 62, 024027 (2000).

[33] S. Iyer and C. M. Will, Phys. Rev. D 35, 3621 (1987).

[34] I. G. Dymnikova, Mod. Phys. D 5, 529 (1996).

[35] I. G. Dymnikova and K. Micha, Phys. Lett. B 685, 1218 (2010).

[36] S. G. Ghosh and S. D. Maharaj, Eur. Phys. J. C 75, 7 (2015).

[37] Z. Y. Fan and X. Wang, Phys. Rev. D 94, 124027 (2016). 
[38] Z. Y. Fan, Eur. Phys. J. C 77, 266 (2017).

[39] D. J. Lovelock, Math. Phys. 12, 498 (1971).

[40] R. C. Myers and J. Z. Simon, Phys. Rev. D 38, 2434 (1988).

[41] S. W. Wei and Y. X. Liu, Phys. Rev. Lett. 115, 111302 (2015).

[42] S. N. Sajadi, N. Riazi and S. H. Hendi, Eur. Phy. J. C 79, 775 (2019).

[43] M. Zhang, J. Jiang and Z. Zhong, Phys. Lett. B 798, 134959 (2019).

[44] D. Glavan and C. Lin, Phys. Rev. Lett. 124, 081301 (2020).

[45] G. T. Horowitz and V. E. Hubeny, Phys. Rev. D 62, 024027 (2000).

[46] C. Ju-Hua and J. W. Yong, Quas. mod. Chin. Phys. B 19 (6), 060401 (2010).

[47] Y. Zhang, E. K. Li and J. L. Geng, Quas. mod. Gen. Relat. 46 (5), 1728 (2014). 